\title{
Regularity to the spherically symmetric compressible Navier-Stokes equations with density-dependent viscosity
}

Lan Huang ${ }^{1 *}$ (D) and Ruxu Lian'

"Correspondence:

huanglan82@hotmail.com

${ }^{1}$ College of Mathematics and

Statistics, North China University of Water Resources and Electric Power

Zhengzhou, P.R. China

\begin{abstract}
This paper is concerned with the dynamics for the compressible Navier-Stokes equations with density-dependent viscosity in bounded annular domains in $R^{3}$. In the paper, we shall analyze the spherical symmetric model and establish the regularity in $H^{2}$ and $H^{4}$ under certain assumptions imposed on the initial data.
\end{abstract}

Keywords: Navier-Stokes equations; Density-dependent viscosity; Regularity; Spherical symmetry

\section{Introduction}

It is well known that the compressible isentropic Navier-Stokes equations which describe the motion of compressible fluids can be written in Eulerian coordinates as

$$
\begin{aligned}
& \rho_{t}+\operatorname{div}(\rho \mathbf{U})=0, \\
& (\rho \mathbf{U})_{t}+\operatorname{div}(\rho \mathbf{U} \otimes \mathbf{U})-\operatorname{div}(\mu(\rho) D(\mathbf{U}))-\nabla(\lambda(\rho) \operatorname{div} \mathbf{U})+\nabla P(\rho)=0,
\end{aligned}
$$

where $\rho(\mathbf{x}, t), \mathbf{U}(\mathbf{x}, t)$ and $P(\rho)=\rho^{\gamma}(\gamma>1)$ stand for the fluid density, velocity and pressure, respectively, and the strain tensor is given by

$$
D(\mathbf{U})=\frac{\nabla \mathbf{U}+(\nabla \mathbf{U})^{T}}{2}
$$

The Lamé viscosity coefficients $\mu(\rho), \lambda(\rho)$ satisfy the natural restrictions

$$
\mu(\rho)>0, \quad \mu(\rho)+N \lambda(\rho) \geq 0 .
$$

For simplicity of the presentation, we consider only the viscosity terms $\mu(\rho)=\rho, \lambda(\rho)=0$ and $D(\mathbf{U})=\nabla \mathbf{U}$. Then (1.1)-(1.2) become

$$
\begin{aligned}
& \rho_{t}+\operatorname{div}(\rho \mathbf{U})=0, \\
& (\rho \mathbf{U})_{t}+\operatorname{div}(\rho \mathbf{U} \otimes \mathbf{U})+\nabla P(\rho)-\operatorname{div}(\rho \nabla \mathbf{U})=0 .
\end{aligned}
$$

(c) The Author(s) 2018. This article is distributed under the terms of the Creative Commons Attribution 4.0 International License (http://creativecommons.org/licenses/by/4.0/), which permits unrestricted use, distribution, and reproduction in any medium, provided you give appropriate credit to the original author(s) and the source, provide a link to the Creative Commons license, and indicate if changes were made. 
We are concerned with the spherically symmetric solutions of system (1.5)-(1.6) in bounded annular domains $G=\left\{\mathbf{x} \in R^{3}, 0<a<|\mathbf{x}|<b<+\infty\right\}$. To this end, we denote

$$
|\mathbf{x}|=r, \quad \rho(\mathbf{x}, t)=\rho(r, t), \quad \mathbf{U}(\mathbf{x}, t)=u(r, t) \frac{\mathbf{x}}{r}
$$

which leads to the following system of equations for $r>0$ :

$$
\begin{aligned}
& \rho_{t}+(\rho u)_{r}+\frac{2 \rho u}{r}=0, \\
& (\rho u)_{t}+\left(\rho u^{2}+\rho^{\gamma}\right)_{r}+\frac{2 \rho u^{2}}{r}-(\rho u)_{r}-\rho\left(\frac{2 u}{r}\right)_{r}=0 .
\end{aligned}
$$

We shall consider problem (1.8)-(1.9) in the region $G$ subject to the initial data

$$
(\rho, u)(r, 0)=\left(\rho_{0}, u_{0}\right)(r), \quad r \in[a, b]
$$

and the boundary condition

$$
u(a, t)=u(b, t)=0, \quad t \in[0, T] .
$$

First we find it convenient to transfer problems (1.8)-(1.11) into that in Lagrangian coordinates and draw the desired results. We introduce the following coordinate transformation:

$$
x(r, t)=\int_{a}^{r} \rho(s, \tau) s^{2} d s, \quad t=\tau,
$$

then the boundaries $r=a$ and $r=b$ become

$$
x=0, \quad x=\int_{a}^{b} \rho(s, \tau) s^{2} d s=\int_{a}^{b} \rho_{0}(s) s^{2} d s,
$$

where $\int_{a}^{b} \rho_{0}(s) s^{2} d s$ is the total initial mass and, without loss of generality, we can normalize it to 1 . So in terms of Lagrangian coordinates, the domain $G$ becomes $\Omega=(0,1)$. The relations between Lagrangian and Eulerian coordinates are satisfied by

$$
\frac{\partial x}{\partial r}=\rho r^{2}, \quad \frac{\partial x}{\partial t}=\rho u r^{2} .
$$

The initial boundary value problem (1.8)-(1.11) are changed to

$$
\begin{aligned}
& \rho_{t}+\rho^{2}\left(r^{2} u\right)_{x}=0, \\
& \frac{u_{t}}{r^{2}}+\left(\rho^{\gamma}\right)_{x}=\left(\rho^{2}\left(r^{2} u\right)_{x}\right)_{x}-\frac{2 \rho_{x} u}{r}, \\
& (\rho, u)(x, 0)=\left(\rho_{0}, u_{0}\right)(x), \quad x \in[0,1], \\
& u(0, t)=u(1, t)=0, \quad t>0 .
\end{aligned}
$$


Much progress was achieved recently on the compressible Navier-Stokes equations with density-dependent viscosity coefficient. Firstly let us recall some well-known results as regards the one-dimensional compressible isentropic Navier-Stokes equations with the flow density being connected with the infinite vacuum, $[19,24,25]$ for the local well-posedness and the global existence of weak solutions to an initial boundary value problem with the viscous gas being connected vacuum states with jump discontinuities, $[9,10]$ for the global behavior with the initial density being piecewise smooth; [28-31,34] for the local existence, the global existence, the asymptotic behavior and the uniqueness of weak solutions with a viscous gas being connected vacuum states with continuous density.

In spatial multi-dimension, there is a huge literature as regards the global existence, the regularity and the asymptotic behavior of a solution to system (1.1)-(1.2) with constant viscosity, we refer the reader to $[2,3,11-14,16,20-23,33]$ and the references therein. For the 3-D flow of a compressible fluid with cylindrical symmetry, the global existence and the large-time behavior of generalized solutions have been proved in $[1,4,5,7,15,23$, $26,27,32]$ for the isentropic and the nonisentropic cases. The corresponding study of the regularity of a solution for any given initial datum has been carried out in [17]. For the 3D flow of compressible fluid with spherical symmetry, there are some interesting results, [18] for the global well-posedness of classical solutions with large oscillations and vacuum; [33] for the global existence and uniqueness of the weak solution without a solid core; [14] for the structure of the solution; [21] for the global existence of the exterior problem and the initial boundary value problem. Besides, we would like to refer to $[6,8]$ as regards the existence and regularity of solutions for micropolar fluid with spherical symmetry in the three-dimensional case.

In the paper, we shall analyze the spherical symmetric model and focus on the initial boundary problem of an isentropic compressible fluid. We show the regularity in $H^{2}$ and $H^{4}$ under certain assumptions imposed on the initial data.

The notation in this paper will be as follows:

$L^{p}, 1 \leq p \leq+\infty, W^{m, p}, m \in N, H^{1}=W^{1,2}, H_{0}^{1}=W_{0}^{1,2}$ denote the usual (Sobolev) spaces on $[0,1]$. To denote various constants, we use $C_{i}(i=1,2,4)$ to denote the generic positive constant depending only on the $H^{i}$ norm of initial datum $\left(\rho_{0}, u_{0}\right)$, $\min _{x \in[0,1]} \rho_{0}(x)$ and variable $t$, respectively. In addition, $\|\cdot\|$ denotes the norm in the space $L^{2}$.

The basic assumption of this paper is the following:

$$
\inf _{[0,1]} \rho_{0}>\underline{\rho},
$$

for some constant $\underline{\rho}>0$.

Theorem 1.1 Let $\gamma>1$. Assume that the initial data $\left(\rho_{0}, u_{0}\right) \in H^{2}(\Omega) \times H^{2}(\Omega)$ and (1.19) hold, then there exists a unique generalized global solution $(\rho(t), u(t)) \in\left(H^{2}(\Omega)\right)^{2}$ to the problem (1.15)-(1.18) verifying that, for any $T>0$,

$$
\begin{aligned}
& \rho \in L^{\infty}\left([0, T], H^{2}(\Omega)\right) \cap L^{2}\left([0, T], H^{2}(\Omega)\right), \\
& u \in L^{\infty}\left([0, T], H^{2}(\Omega)\right) \cap L^{2}\left([0, T], H^{3}(\Omega)\right), \\
& u_{t} \in L^{\infty}\left([0, T], L^{2}(\Omega)\right) \cap L^{2}\left([0, T], H^{1}(\Omega)\right) .
\end{aligned}
$$


Theorem 1.2 Let $\gamma>1$. Assume that the initial data satisfies $(1.19)$ and $\left(\rho_{0}, u_{0}\right) \in H^{4}(\Omega) \times$ $H^{4}(\Omega)$, then there exists a unique generalized global solution $(\rho(t), u(t)) \in\left(H^{4}(\Omega)\right)^{2}$ to the problem (1.15)-(1.18) verifying that, for any $T>0$,

$$
\begin{aligned}
& \rho \in L^{\infty}\left([0, T], H^{4}(\Omega)\right) \cap L^{2}\left([0, T], H^{4}(\Omega)\right), \\
& u \in L^{\infty}\left([0, T], H^{4}(\Omega)\right) \cap L^{2}\left([0, T], H^{4}(\Omega)\right), \\
& u_{t} \in L^{\infty}\left([0, T], H^{2}(\Omega)\right) \cap L^{2}\left([0, T], H^{3}(\Omega)\right), \\
& u_{t t} \in L^{\infty}\left([0, T], L^{2}(\Omega)\right) \cap L^{2}\left([0, T], H^{1}(\Omega)\right) .
\end{aligned}
$$

Corollary 1.1 Under assumptions of Theorem 1.2, (1.23)-(1.24) imply $(\rho(t), u(t))$ is the classical solution verifying that, for any $t>0$,

$$
\|\rho(t)\|_{C^{3+1 / 2}}+\|u(t)\|_{C^{3+1 / 2}} \leq C_{4} .
$$

\section{Proof of Theorem 1.1}

This section is devoted to deriving the estimates of the solutions to prove Theorem 1.1 which will be presented in a sequence of lemmas. We begin with the following lemma.

Lemma 2.1 (Theorem 2.2 in [21]) Under the assumptions in Theorem 1.1, then there exist positive constants $\rho_{*}>0$ and $\rho^{*}>0$ so that the unique global solution $(\rho(t), u(t))$ to problem (1.15)-(1.18) exists and satisfies, for any $T>0$,

$$
\begin{aligned}
& 0<\rho_{*} \leq \rho(x, t) \leq \rho^{*}, \\
& \int_{0}^{1}\left(u^{2}+(\rho-\bar{\rho})^{2}+u_{x}^{2}+u_{t}^{2}+\rho_{x}^{2}\right)(x, t) d x+\int_{0}^{t} \int_{0}^{1}\left(\rho_{x}^{2}+u_{x}^{2}\right. \\
& \left.\quad+u_{t}^{2}+u_{x x}^{2}+u_{x t}^{2}\right)(x, s) d x d s \leq C_{1}, \quad t \in[0, T],
\end{aligned}
$$

where $\bar{\rho}=\frac{1}{b-a} \int_{a}^{b} \rho(s, t) s^{2} d s$.

Lemma 2.2 Under the conditions in Theorem 1.1, for any $T>0$,

$$
\begin{aligned}
& \left\|\rho_{x x}(t)\right\|^{2}+\int_{0}^{t}\left\|\rho_{x x}(s)\right\|^{2} d s \leq C_{2}, \quad t \in[0, T], \\
& \left\|u_{x x}(t)\right\|^{2}+\int_{0}^{t} \int_{0}^{1} u_{x x x}^{2}(x, s) d x d s \leq C_{2}, \quad t \in[0, T] .
\end{aligned}
$$

Proof We infer from (1.16) that

$$
\frac{u_{t}}{r^{2}}=-\gamma \rho^{\gamma-1} \rho_{x}+2 \rho \rho_{x}\left(r^{2} u\right)_{x}+\rho^{2}\left(r^{2} u_{x x}+4 r r_{x} u_{x}+2 r_{x}^{2} u+2 r r_{x x} u\right)-\frac{2 \rho_{x} u}{r} .
$$

Multiplying (2.5) by $u_{x x}$ in $L^{2}(\Omega)$, we deduce

$$
\begin{aligned}
\int_{0}^{1} \rho^{2} r^{2} u_{x x}^{2} d x= & \int_{0}^{1} u_{x x}\left(\frac{u_{t}}{r^{2}}+\gamma \rho^{\gamma-1} \rho_{x}-2 \rho \rho_{x}\left(r^{2} u\right)_{x}\right. \\
& \left.-\rho^{2}\left(4 r r_{x} u_{x}+2 r_{x}^{2} u+2 r r_{x x} u\right)+\frac{2 \rho_{x} u}{r}\right) d x .
\end{aligned}
$$


Using Young's inequality, Sobolev's embedding theorem, and Lemma 2.1, we deduce from (2.6) that

$$
\begin{aligned}
\int_{0}^{1} u_{x x}^{2} d x & \leq C_{1} \int_{0}^{1}\left(u_{t}^{2}+\rho_{x}^{2}+\rho_{x}^{2} u_{x}^{2}\right) d x+\frac{1}{4} \int_{0}^{1} u_{x x}^{2} d x \\
& \leq C_{2}+C_{1}\left\|u_{x}\right\|_{L^{\infty}}^{2} \int_{0}^{1} \rho_{x}^{2}+\frac{1}{4} \int_{0}^{1} u_{x x}^{2} d x \\
& \leq C_{2}+\frac{1}{2} \int_{0}^{1} u_{x x}^{2} d x,
\end{aligned}
$$

whence

$$
\int_{0}^{1} u_{x x}^{2} d x \leq C_{2}
$$

Differentiating (1.16) with respect to $x$, and exploiting (1.15), we have

$$
\left(\frac{u_{t}}{r^{2}}\right)_{x}=\left(-\rho^{\gamma}+\rho^{2}\left(r^{2} u\right)_{x}\right)_{x x}-\left(\frac{2 \rho_{x} u}{r}\right)_{x}
$$

which gives

$$
\rho_{t x x}+\gamma \rho^{\gamma-1} \rho_{x x}=E_{0}(x, t)
$$

with

$$
E_{0}(x, t)=\frac{2 \rho_{x} u r_{x}}{r^{2}}-\frac{2 \rho_{x x} u+2 \rho_{x} u_{x}}{r}-\gamma(\gamma-1) \rho^{\gamma-2} \rho_{x}^{2}+\frac{2 u_{t} r_{x}}{r^{3}}-\frac{u_{t x}}{r^{2}} .
$$

Multiplying (2.9) by $\rho_{x x}$, integrating the resultant over [0,1], we deduce

$$
\begin{aligned}
& \frac{d}{d t}\left\|\rho_{x x}(t)\right\|^{2}+\int_{0}^{1} \gamma \rho^{\gamma-1} \rho_{x x}^{2} d x \\
& \quad=\int_{0}^{1}\left(\frac{2 \rho_{x} u r_{x}}{r^{2}}-\frac{2 \rho_{x x} u+2 \rho_{x} u_{x}}{r}-\gamma(\gamma-1) \rho^{\gamma-2} \rho_{x}^{2}+\frac{2 u_{t} r_{x}}{r^{3}}-\frac{u_{t x}}{r^{2}}\right) \rho_{x x} d x \\
& \quad \leq C_{1}\left\|\rho_{x x}\right\|\left(\left\|u_{t x}\right\|+\left\|u_{t} r_{x}\right\|+\left\|\rho_{x}^{2}\right\|+\left\|\rho_{x} u_{x}\right\|+\left\|\rho_{x} u r_{x}\right\|\right)-2 \int_{0}^{1} \frac{u}{r} \rho_{x x}^{2} d x .
\end{aligned}
$$

Using the Young inequality and the interpolation inequality to the above inequality, then we get

$$
\begin{aligned}
\frac{d}{d t}\left\|\rho_{x x}(t)\right\|^{2} \leq & C_{1}\left(\left\|u_{t x}\right\|^{2}+\left\|u_{t}\right\|^{2}+\left\|\rho_{x}\right\|^{2}+\left\|u_{x}\right\|^{2}+\|u\|^{2}\right) \\
& +C_{1}\left\|\rho_{x x}\right\|^{2}\left(1+\|u\|_{L^{\infty}}^{2}\right) .
\end{aligned}
$$

Integrating (2.10) with respect to $t$, using initial condition $\rho_{0} \in H^{2}$ and Lemma 2.1, we have

$$
\left\|\rho_{x x}(t)\right\|^{2} \leq C_{2}+C_{1} \int_{0}^{t}\left\|\rho_{x x}(s)\right\|^{2} d s, \quad \forall t \in[0, T] .
$$

Then using the Gronwall inequality to the above inequality, we can get (2.3). 
Differentiating (1.16) with respect to $x$, we can obtain

$$
\begin{aligned}
u_{x x x}= & -\frac{1}{\rho^{2} r^{2}}\left[\left(4 r u \rho_{x}^{2} r_{x}+2 r^{2} \rho_{x}^{2} u_{x}+2 \rho \rho_{x x}\left(2 r r_{x} u+r^{2} u_{x}\right)+4 \rho \rho_{x}\left(2 r_{x}^{2} u\right.\right.\right. \\
& \left.+4 r r_{x} u_{x}+2 r r_{x x} u+r^{2} u_{x x}\right)+\rho^{2}\left(6 r_{x} r_{x x} u+6 r_{x}^{2} u_{x}+6 r r_{x x} u+r^{2} u_{x x}\right) \\
& +\rho^{2}\left(6 r_{x} r_{x x} u+6 r_{x}^{2} u_{x}+6 r r_{x x} u_{x}+6 r r_{x} u_{x x}+2 r r_{x x x} u\right)+\frac{2 \rho_{x x} u+2 \rho_{x} u_{x}}{r} \\
& \left.-\frac{2 \rho_{x} u r_{x}}{r^{2}}+\frac{u_{t x}}{r^{2}}-\frac{u_{t} r_{x}}{r^{3}}+\gamma \rho^{\gamma-1} \rho_{x x}+\gamma(\gamma-1) \rho^{\gamma-2} \rho_{x}^{2}\right] .
\end{aligned}
$$

Integrating (2.11) over $x$ and $t$, applying the embedding theorem, Lemma 2.1, (2.7) and (2.3), we conclude, for any $t \in[0, T]$,

$$
\begin{aligned}
\int_{0}^{t} \int_{0}^{1} u_{x x x}^{2} d x d s & \leq C_{1} \int_{0}^{t} \int_{0}^{1}\left(u_{t x}^{2}+\rho_{x}^{4}+\rho_{x x}^{2}+\rho_{x}^{4} u_{x}^{2}+\rho_{x}^{2} u_{x}^{2}+\rho_{x x}^{2} u_{x}^{2}+u_{t}^{2}\right)(x, s) d x d s \\
& \leq C_{1}+C_{1} \int_{0}^{t}\left\|u_{x}\right\|_{L^{\infty}}^{2} \int_{0}^{1}\left(\rho_{x x}^{2}+\rho_{x}^{4}\right) d x d s+C_{1} \int_{0}^{t}\left\|\rho_{x}\right\|_{L^{\infty}}^{2}\left\|u_{x x}\right\|^{2} d s \\
& \leq C_{2}
\end{aligned}
$$

which, along with (2.7), gives (2.4). The proof is complete.

Proof of Theorem 1.1 Clearly, (1.20)-(1.22) follow from Lemmas 2.1-2.2. This completes the proof of Theorem 1.1.

\section{Proof of Theorem 1.2}

In this section, we shall complete the proof of Theorem 1.2. To this end, we assume that in this section that all assumptions in Theorem 1.2 hold. We begin with the following lemma.

Lemma 3.1 The following estimate holds for any $T>0$ :

$$
\left\|u_{t t}(t)\right\|^{2}+\int_{0}^{t}\left\|u_{t t x}(s)\right\|^{2} d s \leq C_{4}+C_{2} \int_{0}^{t}\left\|u_{t x x}(s)\right\|^{2} d s, \quad t \in[0, T]
$$

Proof We easily infer from (1.16) and (2.1)-(2.4) that

$$
\left\|u_{t}(t)\right\| \leq C_{1}\left(\left\|\rho_{x}(t)\right\|+\left\|u_{x}(t)\right\|+\left\|u_{x x}(t)\right\|\right)
$$

Differentiating (1.16) with respect to $x$ and using Lemmas 2.1-2.2, we have

$$
\left\|u_{t x}(t)\right\| \leq C_{2}\left(\left\|\rho_{x}(t)\right\|+\left\|\rho_{x x}(t)\right\|+\left\|u_{x}(t)\right\|+\left\|u_{x x}(t)\right\|+\left\|u_{x x x}(t)\right\|\right),
$$

or

$$
\left\|u_{x x x}(t)\right\| \leq C_{2}\left(\left\|\rho_{x}(t)\right\|+\left\|\rho_{x x}(t)\right\|+\left\|u_{x}(t)\right\|+\left\|u_{x x}(t)\right\|+\left\|u_{t x}(t)\right\|\right) .
$$


Differentiating (1.16) with respect to $x$ twice and using the Cauchy-Schwarz inequality, we have

$$
\begin{aligned}
& \left\|u_{t x x}(t)\right\| \leq C_{2}\left(\left\|\rho_{x}(t)\right\|_{H^{2}}+\left\|u_{x}(t)\right\|_{H^{3}}\right), \\
& \left\|u_{x x x x}(t)\right\| \leq C_{2}\left(\left\|\rho_{x}(t)\right\|_{H^{2}}+\left\|u_{x}(t)\right\|_{H^{2}}+\left\|u_{t x x}(t)\right\|\right) .
\end{aligned}
$$

After differentiating (1.16) with respect to $t$, using (3.2)-(3.3) and (3.5), we can get

$$
\begin{aligned}
\left\|u_{t t}(t)\right\| & \leq C_{2}\left(\left\|u_{t x}(t)\right\|+\left\|u_{t x x}(t)\right\|+\left\|u_{t}(t)\right\|+\left\|\rho_{x}(t)\right\|+\left\|u_{x}(t)\right\|+\left\|u_{x x}(t)\right\|\right) \\
& \leq C_{2}\left(\left\|\rho_{x}(t)\right\|_{H^{2}}+\left\|u_{x}(t)\right\|_{H^{3}}\right) .
\end{aligned}
$$

Now differentiating (1.16) with respect to $t$ twice, multiplying the resulting equation by $\left(\frac{u}{r^{2}}\right)_{t t}$ in $L^{2}(\Omega)$, and using integration by parts and (1.18), we conclude

$$
\begin{aligned}
\frac{1}{2} \frac{d}{d t} \int_{0}^{1}\left(\frac{u}{r^{2}}\right)_{t t}^{2} d x= & -\int_{0}^{1}\left(-\rho^{\gamma}+\rho^{2}\left(r^{2} u\right)_{x}\right)_{t t}\left(\frac{u}{r^{2}}\right)_{t t x} d x \\
& -\int_{0}^{1}\left(\frac{2 \rho_{x} u}{r}-\frac{u^{2}}{r^{3}}\right)_{t t}\left(\frac{u}{r^{2}}\right)_{t t} d x=: A_{1}+A_{2} .
\end{aligned}
$$

We use (2.1)-(2.4), (3.2)-(3.8), (1.16) and the interpolation inequality to deduce that, for any small $\varepsilon \in(0,1)$,

$$
\begin{aligned}
A_{1}= & -\int_{0}^{1}\left(-\rho^{\gamma}+\rho^{2}\left(r^{2} u\right)_{x}\right)_{t t}\left(\frac{u}{r^{2}}\right)_{t t x} d x \\
\leq & -\int_{0}^{1} \rho^{2} u_{t t x}^{2} d x+C_{2}\left(\left\|\rho_{t t} u_{x}\right\|+\left\|\rho_{t}^{2} u_{x}\right\|+\left\|\rho_{t} u_{t}\right\|+\left\|\rho_{t} u_{x}\right\|+\left\|\rho_{t} u_{x t}\right\|+\left\|u_{x}\right\|+\left\|u_{t}\right\|\right. \\
& \left.+\left\|u_{x} u_{t}\right\|+\left\|u_{t x}\right\|+\left\|u_{t t}\right\|\right)\left(\left\|u_{t t x}\right\|+\left\|u_{t t}\right\|+\left\|u_{x} u_{t}\right\|+\left\|u_{t x}\right\|+\left\|u_{t}\right\|+\left\|u_{x}\right\|+\left\|u^{3}\right\|\right) \\
\leq & -\int_{0}^{1} \rho^{2} u_{t t x}^{2} d x+\varepsilon\left\|u_{t t x}(t)\right\|^{2}+C_{2}\left(\left\|\rho_{t t}(t)\right\|_{H^{1}}^{2}+\left\|\rho_{t}(t)\right\|^{2}\right. \\
& \left.+\left\|u_{t}(t)\right\|_{H^{1}}^{2}+\left\|u_{t t}(t)\right\|^{2}+\left\|u_{x}(t)\right\|_{H^{1}}^{2}+\left\|\rho_{x}(t)\right\|_{H^{1}}^{2}\right) \\
\leq & -C_{2}^{-1}\left\|u_{t t x}(t)\right\|^{2}+C_{2}\left(\left\|u_{x}(t)\right\|_{H^{2}}^{2}+\left\|\rho_{x}(t)\right\|_{H^{1}}^{2}+\left\|u_{t}(t)\right\|_{H^{2}}^{2}\right) \\
A_{2}= & -\int_{0}^{1}\left(\frac{2 \rho_{x} u}{r}-\frac{u^{2}}{r^{3}}\right)_{t t}\left(\frac{u}{r^{2}}\right)_{t t} d x \\
\leq & C_{2}\left(\left\|\rho_{t t x}\right\|+\left\|\rho_{t x} u_{t}\right\|+\left\|\rho_{x} u_{t t}\right\|+\left\|\rho_{t x} u^{2}\right\|+\left\|u_{t}^{2}\right\|+\left\|u_{t t}\right\|+\left\|u_{t}\right\|+\left\|\rho_{x t} u\right\|\right. \\
& \left.+\left\|\rho_{x} u_{t}\right\|+\left\|\rho_{x} u^{3}\right\|+\left\|u_{t}\right\|+\left\|u^{3}\right\|\right)\left(\left\|u_{t t}\right\|+\left\|u_{t}\right\|+\left\|u_{t} r_{t}\right\|+\left\|u r_{t t}\right\|+\left\|u r_{t}^{2}\right\|\right) \\
\leq & C_{2}\left(\left\|u_{t}(t)\right\|^{2}+\left\|u_{t t}(t)\right\|^{2}+\left\|u_{t x x}(t)\right\|^{2}+\left\|u_{t x}(t)\right\|^{2}+\left\|\rho_{x}(t)\right\|^{2}+\left\|u_{x}(t)\right\|^{2}\right)
\end{aligned}
$$

Integrating (3.9) with respect to $t$, and using (2.1)-(2.4), initial condition (1.16) and (3.10)(3.11), then we obtain (3.1).

Lemma 3.2 For any $T>0$ and $\varepsilon \in(0,1)$, the following estimate holds:

$$
\left\|u_{t x}(t)\right\|^{2}+\int_{0}^{t}\left\|u_{t x x}(s)\right\|^{2} \leq C_{4}+C_{2}^{-1} \varepsilon^{2} \int_{0}^{t}\left\|u_{t t x}(s)\right\|^{2} d s, \quad t \in[0, T] .
$$


Proof Differentiating (1.16) with respect to $t$ and $x$, then multiplying the resultant by $\left(\frac{u}{r^{2}}\right)_{t x}$ in $L^{2}[0,1]$, and integrating by parts, we know that

$$
\begin{aligned}
\frac{1}{2} & \frac{d}{d t} \int_{0}^{1}\left(\frac{u}{r}\right)_{t x}^{2} d x \\
= & \left.\left(-\rho^{\gamma}+\rho^{2}\left(r^{2} u\right)_{x}\right)_{t x}\left(\frac{u}{r^{2}}\right)_{t x}\right|_{0} ^{1}-\int_{0}^{1}\left(-\rho^{\gamma}+\rho^{2}\left(r^{2} u\right)_{x}\right)_{t x}\left(\frac{u}{r^{2}}\right)_{t x x} d x \\
& \quad+\int_{0}^{1}\left(\frac{u^{2}}{r^{3}}-\frac{2 \rho_{x} u}{r}\right)_{t x}\left(\frac{u}{r^{2}}\right)_{t x} d x \\
= & B_{0}(x, t)+B_{1}(t)+B_{2}(t)
\end{aligned}
$$

where

$$
\begin{aligned}
& B_{0}(x, t)=\left.\left(-\rho^{\gamma}+\rho^{2}\left(r^{2} u\right)_{x}\right)_{t x}\left(\frac{u}{r^{2}}\right)_{t x}\right|_{0} ^{1}, \\
& B_{1}(t)=-\int_{0}^{1}\left(-\rho^{\gamma}+\rho^{2}\left(r^{2} u\right)_{x}\right)_{t x}\left(\frac{u}{r^{2}}\right)_{t x x} d x, \\
& B_{2}(t)=\int_{0}^{1}\left(\frac{u^{2}}{r^{3}}-\frac{2 \rho_{x} u}{r}\right)_{t x}\left(\frac{u}{r^{2}}\right)_{t x} d x .
\end{aligned}
$$

Now using Young's inequality several times, and employing (2.1)-(2.4) and the interpolation inequality, after some calculation, we have, for any $\varepsilon \in(0,1)$,

$$
\begin{aligned}
B_{0}(x, t) \leq & C_{2}\left(\left\|\rho_{t x}\right\|_{L^{\infty}}+\left\|\rho_{t} \rho_{x}\right\|_{L^{\infty}}+\left\|\rho_{x} \rho_{t} u_{x}\right\|_{L^{\infty}}+\left\|\rho_{t x} u_{x}\right\|_{L^{\infty}}+\left\|\rho_{t} u_{x x}\right\|_{L^{\infty}}\right. \\
& \left.+\left\|u_{t x x}\right\|_{L^{\infty}}+\left\|u_{t x}\right\|_{L^{\infty}}\right)\left\|u_{t x}\right\|_{L^{\infty}} \\
\leq & C_{2}\left(\left\|u_{x}\right\|_{H^{2}}+\left\|\rho_{x}\right\|_{H^{1}}+\left\|u_{t x}\right\|^{\frac{1}{2}}\left\|u_{t x x}\right\|^{\frac{1}{2}}+\left\|u_{t x x}\right\|^{\frac{1}{2}}\left\|u_{t x x x}\right\|^{\frac{1}{2}}\right)\left\|u_{t x}\right\|^{\frac{1}{2}}\left\|u_{t x x}\right\|^{\frac{1}{2}} \\
\leq & C_{2}^{-1} \varepsilon^{2}\left(\left\|u_{t x x}\right\|^{2}+\left\|u_{t x x x}\right\|^{2}\right)+C_{2} \varepsilon^{-6}\left(\left\|u_{t x}\right\|^{2}+\left\|u_{x}\right\|_{H^{2}}+\left\|\rho_{x}\right\|_{H^{1}}\right) \\
B_{1}(t) \leq & -\int_{0}^{1} \rho^{2} u_{t x x}^{2} d x+\varepsilon \int_{0}^{1} \rho^{2} u_{t x x}^{2} d x+C_{2}\left(\left\|\rho_{t x}(t)\right\|_{H^{1}}^{2}+\left\|\rho_{x}(t)\right\|_{H^{1}}^{2}\right. \\
& \left.+\left\|u_{x}(t)\right\|_{H^{1}}^{2}+\left\|u_{t}(t)\right\|^{2}+\left\|u_{t x}(t)\right\|^{2}\right) \\
B_{2}(t) \leq & C_{2}\left(\left\|u_{x} u_{t}\right\|+\left\|u_{t x}\right\|+\left\|u_{x}\right\|+\left\|u_{t}\right\|+\left\|u^{3}\right\|+\left\|\rho_{t x x}\right\|+\left\|\rho_{t x} u_{x}\right\|+\left\|\rho_{x x} u_{t}\right\|\right. \\
& \left.+\left\|\rho_{x} u_{t x}\right\|+\left\|\rho_{x x}\right\|+\left\|\rho_{x} u_{x}\right\|+\left\|\rho_{t x}\right\|+\left\|\rho_{x} u_{t}\right\|+\left\|\rho_{x}\right\|\right)\left(\left\|u_{t x}\right\|+\left\|u_{x}\right\|\right) \\
\leq & C_{2}\left(\left\|u_{x}\right\|_{H^{1}}^{2}+\left\|u_{t}\right\|_{H^{1}}^{2}+\left\|\rho_{x}\right\|_{H^{1}}^{2}+\left\|\rho_{t x}\right\|^{2}+\left\|\rho_{t x x}\right\|^{2}\right) \\
\leq & C_{2}\left(\left\|u_{x}\right\|_{H^{2}}^{2}+\left\|u_{t}\right\|_{H^{1}}^{2}+\left\|\rho_{x}\right\|_{H^{1}}^{2}\right)
\end{aligned}
$$

Differentiating (1.16) with respect to $x$ and $t$, and using Lemmas 2.1-2.2 and (3.7)-(3.8), we conclude

$$
\left\|u_{t x x x}(t)\right\| \leq C_{2}\left(\left\|u_{x}(t)\right\|_{H^{2}}+\left\|\rho_{x}(t)\right\|_{H^{1}}+\left\|u_{t x}(t)\right\|_{H^{1}}+\left\|u_{t t x}(t)\right\|\right) .
$$

We integrate (3.13) with respect to $t$, use (3.3), (3.14)-(3.16) and Lemmas 2.1-2.2 to obtain (3.12). The proof is complete. 
Lemma 3.3 The following estimates hold for any $T>0$ :

$$
\begin{aligned}
& \left\|u_{t t}(t)\right\|^{2}+\left\|u_{t x}(t)\right\|^{2}+\int_{0}^{t}\left(\left\|u_{t x x}\right\|^{2}+\left\|u_{t t x}\right\|^{2}\right)(s) \leq C_{4}, \quad t \in[0, T] \\
& \left\|\rho_{x x x}(t)\right\|^{2}+\left\|u_{x x x}(t)\right\|^{2}+\int_{0}^{t}\left(\left\|\rho_{x x x}\right\|^{2}+\left\|u_{x x x x}\right\|^{2}\right)(s) d s \leq C_{4}, \quad t \in[0, T]
\end{aligned}
$$

Proof We insert (3.1) into (3.12) and pick $\varepsilon$ small enough to get (3.18).

Differentiating (2.9) with respect to $x$, we have

$$
\rho_{t x x x}+\gamma \rho^{\gamma-1} \rho_{x x x}=E_{1}(x, t)
$$

where

$$
E_{1}(x, t)=E_{0 x}(x, t)-\gamma(\gamma-1) \rho^{\gamma-2} \rho_{x} \rho_{x x} .
$$

Taking into account estimate (3.18), from (2.1)-(2.4) we can get

$$
\begin{aligned}
\left\|E_{1}(t)\right\| \leq & C_{2}\left(\left\|E_{0 x}\right\|+\left\|\rho_{x} \rho_{x x}\right\|\right) \\
\leq & C_{2}\left(\left\|\rho_{x} u_{x}\right\|+\left\|\rho_{x x}\right\|+\left\|\rho_{x x} u_{x}\right\|+\left\|\rho_{x x x}\right\|+\left\|\rho_{x} u_{x x}\right\|+\left\|\rho_{x x} \rho_{x}\right\|\right. \\
& \left.+\left\|u_{t x}\right\|+\left\|u_{t} \rho_{x}\right\|+\left\|u_{t x x}\right\|\right) \\
\leq & C_{2}\left(\left\|\rho_{x}(t)\right\|_{H^{2}}+\left\|u_{x}(t)\right\|_{H^{2}}+\left\|u_{t}(t)\right\|_{H^{2}}\right)
\end{aligned}
$$

which, along with Lemmas $2.1-2.2$ and (3.18), implies

$$
\int_{0}^{t}\left\|E_{1}(s)\right\|^{2} d s \leq C_{4}+C_{2} \int_{0}^{t}\left\|\rho_{x x x}(s)\right\|^{2} d s .
$$

After multiplying (3.20) by $\rho_{x x x}$ in $L^{2}[0,1]$, we deduce

$$
\frac{1}{2} \frac{d}{d t}\left\|\rho_{x x x}\right\|^{2}+\gamma \int_{0}^{1} \rho^{\gamma-1} \rho_{x x x}^{2} d x \leq C_{1}\left\|E_{1}(t)\right\|^{2}
$$

which implies

$$
\frac{d}{d t}\left\|\rho_{x x x}\right\|^{2} \leq C_{1}\left\|E_{1}(t)\right\|^{2} .
$$

Integrating (3.23) with respect to $t$, using (3.21), we conclude

$$
\left\|\rho_{x x x}\right\|^{2} \leq C_{4}+C_{2} \int_{0}^{t}\left\|\rho_{x x x}(s)\right\|^{2} d s
$$

which, after applying the Gronwall inequality and (3.22), yields

$$
\left\|\rho_{x x x}(t)\right\|^{2}+\int_{0}^{t}\left\|\rho_{x x x}(s)\right\|^{2} d s \leq C_{4}, \quad \forall t \in[0, T] .
$$


By (3.4), (3.6), (3.18) and Lemmas 2.1-2.2, we conclude

$$
\left\|u_{x x x}(t)\right\|^{2}+\int_{0}^{t}\left\|u_{x x x x}(s)\right\|^{2} d s \leq C_{4}, \quad \forall t \in[0, T]
$$

which, along with (3.24), gives (3.19). The proof is complete.

Lemma 3.4 The following estimates hold for any $T>0$ :

$$
\begin{aligned}
& \left\|\rho_{x x x x}(t)\right\|^{2}+\int_{0}^{t}\left\|\rho_{x x x x}(s)\right\|^{2} d s \leq C_{4}, \quad t \in[0, T], \\
& \left\|u_{t x x}(t)\right\|^{2}+\left\|u_{x x x x}(t)\right\|^{2}+\int_{0}^{t}\left\|u_{x x x x x}(s)\right\|^{2} d s \leq C_{4}, \quad t \in[0, T] .
\end{aligned}
$$

Proof Differentiating (1.16) with respect to $t$, and using Lemmas 2.1-2.2 and Lemma 3.3, we can get

$$
\begin{aligned}
\left\|u_{t x x}(t)\right\| & \leq C_{1}\left(\left\|u_{t}(t)\right\|_{H^{1}}+\left\|\rho_{x}(t)\right\|+\left\|u_{x}(t)\right\|_{H^{1}}+\left\|u_{t t}(t)\right\|\right) \\
& \leq C_{4}, \quad \forall t \in[0, T] .
\end{aligned}
$$

Differentiating (3.20) with respect to $x$, we have

$$
\rho_{\text {txxxx }}+\gamma \rho^{\gamma-1} \rho_{x x x x}=E_{2}(x, t),
$$

where

$$
E_{2}(x, t)=E_{1 x}(x, t)-\gamma(\gamma-1) \rho_{x} \rho_{x x x}
$$

and

$$
E_{1 x}(x, t)=E_{0 x x}(x, t)-\gamma(\gamma-1)\left(\rho^{\gamma-2} \rho_{x} \rho_{x x}\right)_{x} .
$$

An easy calculation with the interpolation inequality, (3.2)-(3.8) and Lemmas 2.1-2.2 and Lemma 3.3 gives

$$
\begin{aligned}
\left\|E_{0 x}(t)\right\| \leq & C_{2}\left(\left\|\rho_{x} u_{x}\right\|+\left\|\rho_{x x x} u_{x}\right\|+\left\|\rho_{x x} u_{x x}\right\|+\left\|\rho_{x}^{3}\right\|+\left\|u_{t x} r_{x}\right\|+\left\|u_{t} r_{x x}\right\|++\left\|u_{t x x}\right\|\right) \\
\leq & C_{2}\left(\left\|\rho_{x}\right\|_{H^{3}}+\left\|u_{x}\right\|_{H^{2}}+\left\|u_{t x}\right\|+\left\|u_{t x x}\right\|\right) \\
\left\|E_{0 x x}(t)\right\| \leq & C_{2}\left(\left\|\rho_{x x x x}\right\|+\left\|\rho_{x x} u_{x}\right\|+\left\|\rho_{x} u_{x x}\right\|+\left\|\rho_{x x x} u_{x}\right\|+\left\|\rho_{x x} u_{x x}\right\|\right. \\
& \left.+\left\|u_{t x x}\right\|+\left\|u_{t x} u_{x}\right\|+\left\|u_{t x x x}\right\|\right) \\
\leq & C_{2}\left(\left\|\rho_{x}\right\|_{H^{3}}+\left\|u_{x}\right\|_{H^{2}}+\left\|u_{t x}\right\|_{H^{2}}\right) \\
\left\|E_{1 x}(t)\right\| \leq & C_{2}\left(\left\|E_{0 x x}(t)\right\|+\left\|\rho_{x}^{2} \rho_{x x}\right\|+\left\|\rho_{x x}^{2}\right\|+\left\|\rho_{x} \rho_{x x x}\right\|\right) \\
\leq & C_{2}\left(\left\|\rho_{x}\right\|_{H^{3}}+\left\|u_{x}\right\|_{H^{2}}+\left\|u_{t x}\right\|_{H^{2}} \|\right) .
\end{aligned}
$$

Taking into account estimate (3.30)-(3.32), from (3.29), we obtain

$$
\left\|E_{2}(t)\right\| \leq C_{4}\left(\left\|u_{x}(t)\right\|_{H^{3}}+\left\|\rho_{x}(t)\right\|_{H^{3}}+\left\|u_{t x}(t)\right\|_{H^{2}}\right)
$$


Inserting (3.17) into (3.33), and integrating (3.33) with respect to $t$, using Lemmas 2.12.2 and Lemma 3.3, we have

$$
\int_{0}^{t}\left\|E_{2}(s)\right\|^{2} d s \leq C_{4}+C_{2} \int_{0}^{t}\left\|\rho_{x x x x}(s)\right\|^{2} d s, \quad \forall t \in[0, T] .
$$

Multiplying (3.28) by $\rho_{x x x x}$ in $L^{2}[0,1]$, we can get

$$
\frac{1}{2} \frac{d}{d t}\left\|\rho_{x x x x}\right\|^{2}+\gamma \int_{0}^{1} \rho^{\gamma-1} \rho_{x x x x}^{2} d x \leq C_{2}\left\|E_{2}(t)\right\|^{2},
$$

which implies

$$
\frac{d}{d t}\left\|\rho_{x x x x}\right\|^{2} \leq C_{2}\left\|E_{2}(t)\right\|^{2} .
$$

Integrating (3.36) with respect to $t$, using (3.34), we conclude

$$
\left\|\rho_{x x x x}\right\|^{2} \leq C_{4}+C_{2} \int_{0}^{t}\left\|\rho_{x x x x}(s)\right\|^{2} d s, \quad t \in[0, T]
$$

which, after using Gronwall's inequality, yields

$$
\left\|\rho_{x x x x}(t)\right\|^{2} \leq C_{4}, \quad t \in[0, T] .
$$

Thus, we can obtain (3.25) by virtue of (3.37)-(3.38).

By (3.6), (3.17), (3.25), Lemmas 2.1-2.2 and Lemma 3.3, we have

$$
\left\|u_{x x x x}(t)\right\|^{2}+\int_{0}^{t}\left\|u_{t x x x}(s)\right\|^{2} d s \leq C_{4}, \quad t \in[0, T] .
$$

On the other hand, we differentiate (1.16) with respect to $x$ three times, use Lemmas 2.12.2 and (3.25), (3.27) to conclude, for any $t \in[0, T]$,

$$
\left\|u_{x x x x x}(t)\right\| \leq C_{4}\left(\left\|u_{t x x x}(t)\right\|+\left\|u_{x}(t)\right\|_{H^{3}}+\left\|\rho_{x}(t)\right\|_{H^{3}}\right) .
$$

Thus we deduce from (3.38)-(3.40) that

$$
\int_{0}^{t}\left\|u_{x x x x x}(s)\right\|^{2} d s \leq C_{4}, \quad \forall t \in[0, T]
$$

which, along with (3.39), gives (3.26). This completes the proof of the lemma.

Proof of Theorem 1.2 Applying Lemmas 2.1-2.2 and Lemmas 3.1-3.4, we readily get estimates (1.23)-(1.26). This completes the proof of Theorem 1.2.

\section{Conclusions}

In this paper, we have established the regularity of global solutions for the spherically symmetric compressible fluid with density-dependent viscosity in $H_{2}$ and $H_{4}$. The biggest difference from other papers is that our domain has spherical symmetry and the viscosity coefficients are density dependent. 


\section{Acknowledgements}

The authors would like to thank the referees for their useful suggestions, which have significantly improved the paper.

\section{Funding}

This research was supported by the NSFC (No. 11501199) and the Natural Science Foundation of Henan Province (No. 18B110010).

\section{Availability of data and materials}

Data sharing not applicable to this article as no datasets were generated or analyzed during the current study.

\section{Competing interests}

The authors declare that they have no competing interests.

Authors' contributions

All authors carried out the proofs and conceived the study. All authors read and approved the final manuscript.

\section{Publisher's Note}

Springer Nature remains neutral with regard to jurisdictional claims in published maps and institutional affiliations.

\section{Received: 27 February 2018 Accepted: 24 May 2018 Published online: 30 May 2018}

\section{References}

1. Aoyama, R., Kagei, Y.: Large time behavior of solutions to the compressible Navier-Stokes equations around a parallel flow in a cylindrical domain. Nonlinear Anal. 127, 362-396 (2015)

2. Bresch, D., Desjardins, B.: Existence of global weak solutions for a $2 \mathrm{D}$ viscous shallow water equations and convergence to the quasi-geostrophic model. Commun. Math. Phys. 238, 211-223 (2003)

3. Bresch, D., Desjardins, B.: On the construction of approximate solutions for the $2 \mathrm{D}$ viscous shallow water model and for compressible Navier-Stokes models. J. Math. Pures Appl. 86, 362-368 (2006)

4. Cui, H., Yao, Z:: Asymptotic behavior of compressible $p$-th power Newtonian fluid with large initial data. J. Differ. Equ. 258, 919-953 (2015)

5. Dražić, I.: 3-D flow of a compressible viscous micropolar fluid with cylindrical symmetry: a global existence theorem. Math. Methods Appl. Sci. 40, 4785-4801 (2017)

6. Dražić, l., Mujaković, N.: 3-D flow of a compressible viscous micropolar fluid with spherical symmetry: a local existence theorem. Bound. Value Probl. 2012,69 (2012)

7. Dražić, l., Mujaković, N., Črnjarić-Žic, N.: Three-dimensional compressible viscous micropolar fluid with cylindrical symmetry: derivation of the model and a numerical solution. Math. Comput. Simul. 140, 107-124 (2017)

8. Dražić, I., Simčić, L., Mujaković, N.: 3-D flow of a compressible viscous micropolar fluid with spherical symmetry: regularity of the solution. J. Math. Anal. Appl. 438(1), 162-183 (2016)

9. Fang, D., Zhang, T:. Global solutions of the Navier-Stokes equations for compressible flow with density-dependent viscosity and discontinuous initial data. J. Differ. Equ. 222, 63-94 (2006)

10. Fang, D., Zhang, T:: Discontinuous solutions of the compressible Navier-Stokes equations with degenerate viscosity coefficient and vacuum. J. Math. Anal. Appl. 318, 224-245 (2006)

11. Feireisl, E.: Dynamics of Viscous Compressible Fluids. Oxford University Press, Oxford (2004)

12. Frid, H., Shelukhin, V.: Boundary layers for the Navier-Stokes equations of compressible fluids. Commun. Math. Phys. 208, 309-330 (1999)

13. Frid, H., Shelukhin, V.V.: Vanishing shear viscosity in the equations of compressible fluids for the flows with the cylinder symmetry. SIAM J. Math. Anal. 31, 1144-1156 (2000)

14. Guo, Z., Li, H., Xin, Z.: Lagrange structure and dynamics for solutions to the spherically symmetric compressible Navier-Stokes equations. Commun. Math. Phys. 209, 371-412 (2012)

15. Huang, L., Dražić, I.: Large-time behavior of solutions to the 3-D flow of a compressible viscous micropolar fluid with cylindrical symmetry. Math. Methods Appl. Sci. To appear

16. Huang, L., Lian, R.: Exponential stability of spherically symmetric solutions for compressible viscous micropolar fluid. J. Math. Phys. 56, 071503 (2015)

17. Huang, L., Lian, R.: Regularity for compressible isentropic Navier-Stokes equations with cylinder symmetry. J. Inequal. Appl. 2016, $117(2016)$

18. Huang, X., Li, J., Xin, Z:: Global well-posedness of classical solutions with large oscillations and vacuum to the three-dimensional isentropic compressible Navier-Stokes equations. Commun. Pure Appl. Math. 65, 549-585 (2012)

19. Jiang, S., Xin, Z., Zhang, P.: Global weak solutions to 1D compressible isentropic Navier-Stokes equations with density-dependent viscosity. Methods Appl. Anal. 12, 239-252 (2005)

20. Jiang, S., Zhang, J.: Boundary layers for the Navier-Stokes equations of compressible heat-conducting flows with cylindrical symmetry. SIAM J. Math. Anal. 41, 237-268 (2009)

21. Lian, R., Huang, L., Liu, J.: Global solutions to the spherically symmetric compressible Navier-Stokes equations with density-dependent viscosity. J. Appl. Math. 2012, Article ID 395209 (2012)

22. Lions, P.L.: Mathematical Topics in Fluids Dynamics, Vol. 2. Compressible Models. Oxford Sci. Publ. Oxford University Press, Oxford (1996)

23. Liu, J., Lian, R.: Global existence of the cylindrically symmetric strong solution to compressible Navier-Stokes equations. Abstr. Appl. Anal. 2014, Article ID 728715 (2014)

24. Liu, T., Xin, Z., Yang, T.: Vacuum states of compressible flow. Discrete Contin. Dyn. Syst. 4, 1-32 (1998)

25. Okada, M., Matusšu-Necčasová, ̌̆., Makino, T.: Free boundary problem for the equation of one-dimensional motion of compressible gas with density-dependent viscosity. Ann. Univ. Ferrara, Sez. 7: Sci. Mat. 48, 1-20 (2002) 
26. Qin, Y.: Exponential stability for the compressible Navier-Stokes equations with the cylinder symmetry in $R^{3}$. Nonlinear Anal., Real World Appl. 11, 3590-3607 (2010)

27. Qin, Y., Jiang, L.: Global existence and exponential stability of solutions in $H^{4}$ for the compressible Navier-Stokes equations with the cylinder symmetry. J. Differ. Equ. 249, 1353-1384 (2010)

28. Vong, S., Yang, T., Zhu, C.: Compressible Navier-Stokes equations with degenerate viscosity coefficient and vacuum (III). J. Differ. Equ. 192, 475-501 (2003)

29. Yang, T., Yao, Z., Zhu, C.: Compressible Navier-Stokes equations with density-dependent viscosity and vacuum. Commun. Partial Differ. Equ. 26, 965-981 (2001)

30. Yang, T., Zhao, H.: A vacuum problem for the one-dimensional compressible Navier-Stokes equations with density-dependent viscosity. J. Differ. Equ. 184, 163-184 (2002)

31. Yang, T., Zhu, C.: Compressible Navier-Stokes equations with degenerate viscosity coefficient and vacuum. Commun Math. Phys. 230, 329-363 (2002)

32. Yao, L., Zhang, T., Zhu, C.: Boundary layers for compressible Navier-Stokes equations with density-dependent cylindrical symmetry. Ann. Inst. Henri Poincaré, Anal. Non Linéaire 28, 677-709 (2011)

33. Zhang, T., Fang, D.: Global behavior of spherically symmetric Navier-Stokes equations with density-dependent viscosity. J. Differ. Equ. 236, 293-341 (2007)

34. Zhu, C.: Asymptotic behavior of compressible Navier-Stokes equations with density-dependent viscosity and vacuum. Commun. Math. Phys. 293, 279-299 (2010)

\section{Submit your manuscript to a SpringerOpen ${ }^{\circ}$ journal and benefit from:}

- Convenient online submission

- Rigorous peer review

Open access: articles freely available online

- High visibility within the field

Retaining the copyright to your article

Submit your next manuscript at $\boldsymbol{~ s p r i n g e r o p e n . c o m ~}$ 